\title{
Demo Abstract: Vehicle Detection and Classification Using Passive Infrared Sensing
}

\author{
Enas Oudat*, Mustafa Mousa*, and Christian Claudel ${ }^{\Psi}$ \\ *King Abdullah University of Science and Technology \\ $\Psi$ The University of Texas at Austin
}

\begin{abstract}
We propose a new sensing device that can simultaneously monitor urban traffic congestion and another phenomenon of interest (flash floods on the present case). This sensing device is based on the combination of an ultrasonic rangefinder with one or multiple remote temperature sensors. We show an implementation of this device, and illustrate its performance in both traffic flow sensing. Field data shows that the sensor can detect vehicles with a $99 \%$ accuracy, in addition to estimating their speed and classifying them in function of their length. The same sensor can also monitor urban water levels with an accuracy of less than $2 \mathrm{~cm}$.

Keywords-Speed estimation, Vehicle classification, Flash flood, Wireless Sensor Networks.
\end{abstract}

\section{INTRODUCTION}

Wireless sensor networks (WSNs) are widely used for monitoring and control applications such as environmental surveillance [3]. We presently focus on sensors that could monitor simultaneously flash floods and traffic congestion in cities. Multiple sensing principles can be thought of in the context of traffic monitoring, including magnetometers [2], traffic cameras [5], acoustic sensors [4], RADAR (Radio Detection And Ranging) or LIDAR (Laser Infrared Detection And Ranging) [1]. These sensing methods are typically expensive, since they require advanced processing circuitry. In contrast, passive infrared sensors (PIRs) and ultrasonic rangefinders are low cost, reliable and small, and can be readily used for both sensing applications. They are particularly suitable for traffic monitoring systems as they can be easily deployed on the road sides or on traffic lights, and can measure without contact with the target. They can also monitor distances, which make them particularly suitable for the present flash flood monitoring application. In this article, we propose a new type of traffic and flash flood sensors based on the combination of ultrasonic rangefinders with one or multiple passive infrared temperature sensors. This sensor combination can be used as a backbone for a dual urban traffic flash flood wireless sensor network, since it can monitor vehicle speeds, counts, density and vehicle types as well as pluviometry, water presence and water level with a relatively high accuracy.

\section{SYSTEM COMPONENTS}

The traffic and flash flood sensor node that we investigate in this article contains six passive infrared sensors and one ultrasonic rangefinder. The presence of multiple passive infrared sensors allows traffic monitoring on a higher number of traffic lanes. The six passive infrared thermopiles are

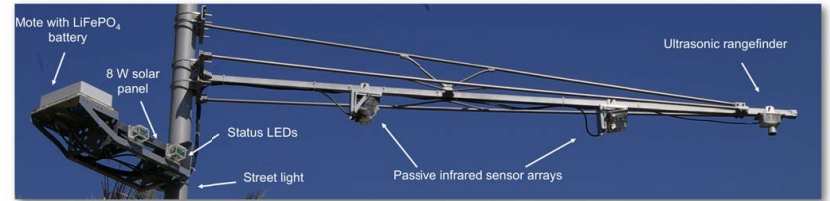

Figure 1. Traffic/Flood sensor node installed on a public street light. Melexis MLX90614 connected to the platform via SMBus. These sensors simultaneously measure the temperature in their field of view and their own temperature. The ultrasonic rangefinder is a MaxBotix MB7066 measuring its distance to below objects. This sensor is connected to the microcontroller (developed by our group for this specific sensing application around a $168 \mathrm{MHz}$ ARM Cortex M4 (STM32F407 from ST Microelectronics), with $192 \mathrm{kB}$ of RAM) via a serial port. In the tested configuration the ultrasonic rangefinder generates data at a $10 \mathrm{~Hz}$ rate, and the measurement frequency of each of the six thermopiles is set at $16 \mathrm{~Hz}$. The measurements are sent wirelessly to a sink node, and are then pushed to an input database for processing.

To mount the sensor in an overhead configuration, we designed, using CAD software, a lightweight aluminum alloy frame that weights less than $6 \mathrm{~kg}$, and can be mounted on regular street lights, extending 3 meters away from the curb side. Four sensors have been deployed to date, including two sensors in a flood prone area. An example sensor is shown in Figure 1.

\section{TRAFFIC MONITORING PERFORMANCE}

Passing vehicles are detected by the passive infrared sensors from the thermal perturbations that they create. The rangefinder can also detect the presence of vehicles below it from the distance measurement timeseries.

\section{A. Vehicle detection using Gaussian Mixture Models}

The detection of vehicles using passive infrared temperature measurements is presently done using Gaussian Mixture models (GMM), in which we classify the data into events, an event corresponding to a passing vehicle. Based on this approach, sensor measurements, $Y^{k}$, are assumed to be a mixture of two Gaussian distributions corresponding to event: $\operatorname{Pr}\left(\right.$ Event $\left./ Y^{k}\right)$ and no event: $\operatorname{Pr}\left(\right.$ No_event $\left./ Y^{k}\right)$.

Figure 2 shows the performance of GMM on a raw temperature dataset generated by the PIR sensors. As can be seen from this Figure, the GMM algorithm allows a reliable and accurate detection of vehicle events, as illustrated in Table I. 
Table I. PERFormance Results of GMM ALGORITHM.

\begin{tabular}{|l|l|l|l|}
\hline Sensor ID & Precision (\%) & Recall (\%) & Accuracy(\%) \\
\hline Sensor 1 & 97.78 & 96.70 & 99.98 \\
\hline Sensor 2 & 94.67 & 95.95 & 99.98 \\
\hline Sensor 3 & 92.80 & 99.15 & 99.97 \\
\hline
\end{tabular}

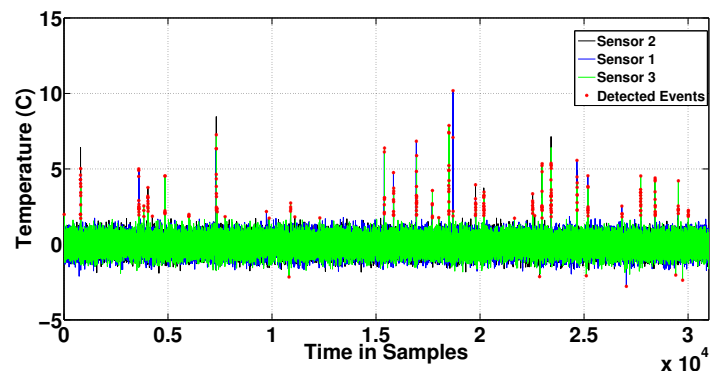

Figure 2. Time series and the detected events by all sensors

\section{B. Speed estimation}

Once the events have been identified by the GMM algorithm, the problem of estimating the vehicle length and speed boils down to an estimation of the delay occurring between the detection by consecutive sensors (each passive infrared sensors covers a given region of the road), and the total duration of the detection. The actual shape of the temperature signal can also be used to infer and classify the vehicle type.

The speed estimation algorithm in particular relies on a constrained cross correlation method in which we search for the maximal correlation occurring during a feasible time period. This time period is constrained by the average performance of the vehicles, and the speed limit of the road. It can be summarized as:

$$
\begin{array}{ll}
\underset{\tau}{\arg \max } & R_{x x(t, t+\tau)} \\
\text { subject to } & \tau_{\min } \succeq \tau_{i} \succeq \tau_{\max },
\end{array}
$$

Estimated delay along with the duration of event are used to estimate the speed and the length of the passing vehicle using Bayesian inference. The performance of the sensor in terms of speed estimation and vehicle length estimation is shown in Figure 3 and Figure 4. As can be seen from these figures, the error in speed estimation is acceptably low (mean error of 5 $\mathrm{kph}$ in speed estimation), and the vehicle lengths are reliably estimated.

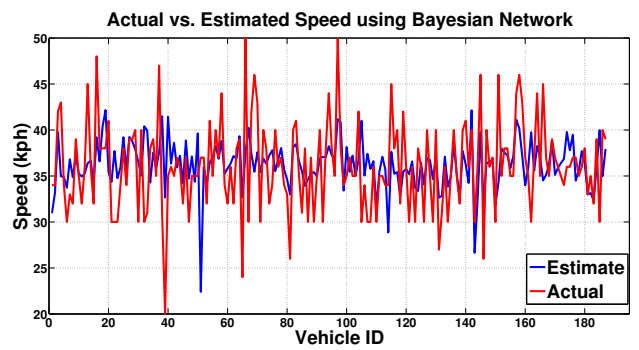

Figure 3. Actual vs. estimated vehicle speed based on Bayesian inference using PIR sensors and ultrasound measurements.

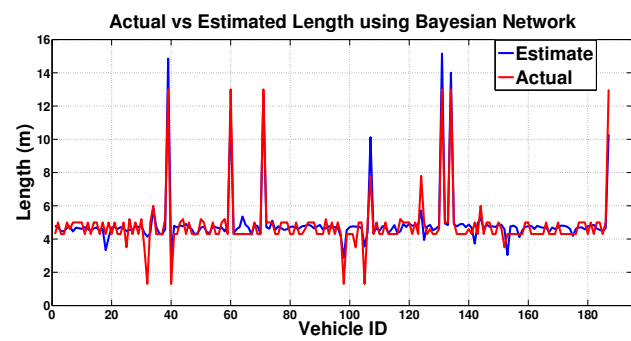

Figure 4. Actual vs. estimated vehicle length based on Bayesian inference using PIR sensors and ultrasound measurements.

Once the speed and the length of the vehicle are estimated, we can use them for vehicle classification using Neural Networks $(\mathrm{NN})$ or Bayesian inference $(\mathrm{BN})$. We have classified the vehicles in five different categories, namely: sedan vehicle, Pick up truck, off-road vehicle, bus, and two wheeler. The classification results are shown in the below table.

Table II. PERFORMANCE RESUlTS OF VEHICLE CLASSIFICATION USING PIR AND ULTRASOUND SENSORS.

\begin{tabular}{|l|l|l|l|c|l|}
\hline \multirow{2}{*}{ Class } & \multicolumn{2}{|c|}{ Recall (\%) } & \multicolumn{2}{c|}{ Precision (\%) } & \multirow{2}{*}{ Num. of Instances } \\
\cline { 2 - 5 } & BN & NN & BN & NN & \\
\hline Sedan vehicle & 94.50 & 92.04 & 99.04 & 86.67 & 109 \\
\hline Pick up truck & 86.96 & 47.83 & 100 & 84.62 & 23 \\
\hline off-road vehicle & 40 & 88.89 & 100 & 86.96 & 43 \\
\hline Bus & 100 & 90.00 & 100 & 90.00 & 8 \\
\hline two wheeler & 100 & 50 & 100 & 40.00 & 4 \\
\hline
\end{tabular}

\section{CONCLUSION AND FUTURE WORK}

In this article, we presented a new type of wireless sensor platform applicable to urban traffic flow and flash flood monitoring, as well as processing algorithms enabling one to monitor both phenomena simultaneously. The proposed sensor comprises an ultrasonic rangefinder and one or multiple passive infrared temperature sensors.

We show that the sensor can accurately monitor traffic flow conditions. Future work will be dedicated to the deployment of a larger number of flash flood/traffic sensor nodes, and their integration within traffic monitoring systems and flash flood monitoring systems, to assess the performance of the complete system (particularly for the flash flood sensing component).

\section{REFERENCES}

[1] G. Alessandretti, A. Broggi, and P. Cerri. Vehicle and guard rail detection using radar and vision data fusion. Intelligent Transportation Systems, IEEE Transactions on, 8(1):95-105, 2007.

[2] C. M. Day, H. Premachandra, T. M. Brennan, J. R. Sturdevant, and D. M. Bullock. Operational evaluation of wireless magnetometer vehicle detectors at signalized intersection. Transportation Research Record: Journal of the Transportation Research Board, 2192(1):11-23, 2010.

[3] A. H. Dehwah, M. Mousa, and C. G. Claudel. Lessons learned on solar powered wireless sensor network deployments in urban, desert environments. Ad Hoc Networks, 28:52-67, 2015.

[4] S. Erb. Classification of vehicles based on acoustic features. na, 2007.

[5] T. N. Schoepflin and D. J. Dailey. Algorithms for calibrating roadside traffic cameras and estimating mean vehicle speed. In Intelligent Transportation Systems Conference, 2007. ITSC 2007. IEEE, pages 277283. IEEE, 2007. 